\title{
The dorsal posterior insula subserves a fundamental role in human pain
}

\author{
Andrew Reilly SegerdahI ${ }^{\# 1,2, \S}$, Melvin Mezue ${ }^{\# 1,2}$, Thomas W. Okell ${ }^{1}$, John T. Farrar ${ }^{3}$, Irene \\ Tracey $^{1,2}$ \\ ${ }^{1}$ Oxford Centre for Functional Magnetic Resonance Imaging of the Brain (FMRIB) \\ ${ }^{2}$ Nuffield Division of Anaesthetics, Nuffield Department of Clinical Neurosciences, University of \\ Oxford, Oxford, UK \\ ${ }^{3}$ Center for Clinical Epidemiology and Biostatistics, School of Medicine, University of \\ Pennsylvania, Philadelphia, PA, USA \\ \# These authors contributed equally to this work.
}

\section{Abstract}

Several brain regions are implicated in human painful experiences but none are proven as specific to pain. Here, we exploit arterial spin-labelling quantitative perfusion imaging and a novel paradigm to identify a specific role for the dorsal posterior insula (dpIns) in pain. Tract tracing studies in animals identify a similar region as fundamental to nociception, which suggests the dpIns is its human homologue and, as such, a potential therapeutic target.

\begin{abstract}
Human neuroimaging studies that measure how nociceptive inputs are encoded to produce pain experiences have yet to identify regional activity specific to pain. Many cortical regions are activated; however, pain is a multifactorial experience encompassing altered attention, anxiety, threat, and many other non-specific features reflected in these activations. Despite extensive study using sophisticated psychological and pharmacological paradigms that aim to disambiguate pain specific from non-specific components, we have yet to identify a painspecific brain region $(1,2,3,4,5,6)$. Part of the difficulty relates to limitations of neuroimaging tools and confounds in paradigm designs.

The aim of the present study is to explore cortical activations that have stimulus response functions mirroring the pain experienced by subjects over several hours in response to a controlled and slowly varying nociceptive input. We hypothesized that regions showing a significant coupling between absolute cerebral blood flow (CBF) and the intensity of tonic pain experienced, as measured using pain intensity ratings over time, are well suited to be candidate pain specific brain regions. To accomplish this, we used an optimized Arterial Spin Labeling (ASL) functional imaging method to quantify cortical activation in response to continuously varying capsaicin-induced heat pain on the right leg in seventeen awake,
\end{abstract}

\footnotetext{
$\S$ To whom correspondence should be addressed: Dr. Andrew Reilly Segerdahl, Centre for Functional Magnetic Resonance Imaging of the Brain (FMRIB), John Radcliffe Hospital, Headington, Oxford, OX3 9DU, United Kingdom, Tel: +44 (0) 1865222 736, Fax: +44(0) 1865222 717, andrew.segerdahl@ndcn.ox.ac.uk.
} 
healthy human subjects. ASL allows us to quantify pain-related neural function in absolute physiological units; therefore, we can reliably interrogate changes in brain activity during the evolution of tonic pain, as it habituates, is experimentally exacerbated and is finally relieved.

The group mean pain intensity ratings for the entire experiment are shown in Figure 1. Results confirm that the onset, maintenance and temperature-driven manipulation of the pain state either by heat exacerbated rekindling or cooling-induced relief was robust and consistent across participants.

Next, we investigated the correlation between absolute $\mathrm{CBF}$ changes and pain ratings over the full experimental time course (Figure 2). From a whole brain perfusion analysis, the only significant positive correlation between absolute CBF changes and pain ratings within subjects was observed in the contralateral dpIns (Figure 2a: Linear regression, Mixed Effects; $\mathrm{z}>2.3, \mathrm{p}<0.01$ ). This highly significant relationship can be further illustrated by the correlation plotted in figure $2 \mathrm{~b}$. Figure $2 \mathrm{c}$ illustrates the close alignment of the dynamic changes in dpIns activity, as measured with $\mathrm{CBF}$, and the pain intensity ratings over the entire experiment.

To validate the pain specificity of our dpIns result, we employed an identical imaging paradigm in a second cohort of subjects (7/12 from original cohort) to that scanned during the initial pain onset phase but using an innocuous, slowly varying vibration stimulus applied to subjects' feet (Supplementary figure 2). A linear regression analysis of CBF with either stimulus intensity or the subjects' ratings of vibratory intensity identified a nonsignificant sub-threshold activation cluster only within the contralateral medial operculum (Supplementary figure 2).

Work by Craig and colleagues using extensive tract tracing and microelectrode work in monkeys have defined a nociceptive specific cortical representation of incoming sensory stimuli that is modality, intensity and location specific. This is within a sub-region of the dorsal posterior insula (dpIns) (7,8). Validation of a homologous structure within humans has yet to occur. Yet, a somatotopy for nociceptive inputs within the posterior insula exists for cutaneous and intramuscular stimuli $(9,10,11)$; intra-cortical recordings in epilepsy patients show that electrical stimulation of this region triggers pain at specific body sites (12) and lesions here alter pain experiences $(13,14,15)$. Therefore, a growing body of literature suggests that a subsection of the posterior insula is both anatomically and functionally well suited to serve a primary and fundamental role in pain processing.

In light of this literature, we overlaid the dpIns cluster identified from our study onto previously published coordinates from the studies described above (Figure 3). There is a striking overlap.

Here, we exploit the benefits of quantitative perfusion neuroimaging to investigate slowly varying neural states highly relevant to complex human perceptions, such as pain. Using this methodology and a novel paradigm and analysis, we have been able to identify the dpIns as subserving a fundamental role in pain and the likely human homologue of the nociceptive region identified from animal studies. Future work targeting dpIns activity might provide a 
window to explore fundamental mechanisms related to how pain emerges from nociception and possibly a novel therapeutic approach in certain chronic pain conditions.

The research was funded by the following organizations who we wish to acknowledge: Medical Research Council of Great Britain and Northern Ireland (FMRIB Centre), the National Institute for Health Research Oxford Biomedical Research Centre, the Wellcome Trust, and the Innovative Medicines Initiative joint undertaking, under Grant Agreement no 115007-resources of which are composed of financial contribution from the European Union's Seventh Framework Programme (FP7/2007-2013) and EFPIA companies' in-kind contribution. We would also like to acknowledge Dr. Falk Eippert, Dr. Katja Wiech, and Dr. Michael Chappell for their insights into the work. The views expressed are those of the author(s) and not necessarily those of the NHS, the NIHR or the Department of Health.

\section{Online Methods}

\section{Participants}

Seventeen healthy subjects (11 female, age [mean \pm s.e.m.] $=24.1 \pm 1.8$ ) were recruited to participate in this study after screening to exclude any history of neurological conditions, regular use of medication, allergies to chilli and/or MRI contraindications. Subjects were asked to avoid caffeine for 6 hours prior to each session. Informed consent was obtained and experimental procedures approved in accordance with the local ethics committee.

\section{Study design}

Subjects were scanned in two phases (separated by 35 mins.) during the same visit. The purpose of this experimental design was to maximize the range of pain intensities associated with the topical capsaicin cream paradigm over a 90-minute period in combination with heating and cooling as further described below. The experimental design did not necessitate subject randomization.

\section{Phase I}

Subjects were scanned at baseline ( 7 minutes) before $1 \%$ capsaicin cream was applied to a $1 \times 3$ inch region on the antero-medial aspect of the lower right leg. The capsaicin cream was held in place with sterile dressing and layered with cloth to maintain a constant skin temperature while scanning. Immediately after capsaicin application, subjects were scanned for an additional 28 minutes to capture the onset (i.e. 'pain onset'; 21 minutes) and peak of the capsaicin pain experience ('pain peak'; 7 minutes).

Online pain ratings were recorded using a visual analogue scale (VAS) (Anchors: No pain, Severe pain) at $\sim 2.5$ minute intervals during each of the pseudo-continuous arterial spin labelling (pCASL) scans-3 times during the baseline block, 7 times during the 'pain onset' block and an additional 3 times during the pain peak. Three subjects did not reach a pain rating of 5/10 after 28 minutes and were therefore scanned for an additional 7 minutes to allow the onset of the peak pain state to unfold.

Following this, subjects were taken out of the MRI scanner and were monitored in a nearby temperature-controlled room for 35 minutes. The capsaicin treated leg was elevated to 
simulate the conditions of the scanner. During this phase, verbal pain ratings (using an 11point numerical rating scale) were taken every 5 minutes.

\section{Phase II}

The second phase of scanning began approximately 35 minutes after the peak of the pain state was scanned. Subjects were scanned using the same perfusion imaging parameters used in Phase I to image the late pain state ('habituation'; 7 minutes), after the application of a warming water bottle to the site of capsaicin cream application ('rekindle'; 7 minutes) and lastly, after application of a cooling water bottle to the capsaicin site ('relief'; 7 minutes).

The hot water was applied at a temperature of $100^{\circ} \mathrm{F}$ (as tested with an infrared thermometer), while the cold water temperature varied between $45-50^{\circ} \mathrm{F}$. The water bottles were placed directly on top of the application region (in the case of the cold water bottle, separated from the skin by a thin layer of cloth to avoid injury). After completion of the second scan phase, the capsaicin cream was removed.

For all scans, when not rating their pain using the VAS scale, subjects were asked to fixate on a cross, which was displayed on a projector.

The external temperature of the right leg around the region of capsaicin application $(2 \mathrm{~cm}$ medial to the application site) was monitored with an infrared thermometer at 5 time points: before capsaicin application, before the end of phase I, before application of the hot water bottle, before application of the cold water bottle and at the end of phase II.

\section{MRI data acquisition}

All subjects were scanned using a Siemens 3T Verio whole-body MR scanner (Erlangen, Germany) equipped with a 32-channel head coil and a body coil.

A time-of-flight MR angiography neck scan was acquired approximately $8 \mathrm{~cm}$ below the Circle of Willis to visualize the brain's feeding arteries. For each subject, the labelling plane was aligned perpendicular to the internal carotid and vertebral arteries. The location of the plane was normalized to a point between the curvatures of the vertebral arteries, where all feeding arteries run perpendicular to the transverse plane. B0 shimming was performed over the imaging region and the labelling plane to minimize off-resonance effects.

We used a pCASL acquisition sequence with background pre-saturation suppression as described recently (17). Images were acquired in separate consecutive blocks, each composing 6 different post-labelling delays: 0.25, 0.5, 0.75, 1, 1.25, 1.5 (seconds). Arterial blood was magnetically tagged using a labelling duration of 1.4 seconds. Other imaging parameters were: single shot echo planar imaging $(\mathrm{EPI})$, repetition time $(\mathrm{TR})=4 \mathrm{~s}$, echo time $(\mathrm{TE})=13 \mathrm{~ms}$, Partial Fourier $=6 / 8$, field of view $(\mathrm{FOV})=220 \times 220$, matrix $=64 \times 64,24$ ascending slices, slice thickness $=4.95 \mathrm{~mm}$, slice acquisition time $=0.0452 \mathrm{~s}$. For each scan, 114 volumes (control and tag) were acquired, corresponding to 7 minutes of scanning. During the 'pain onset' phase, the scanner was run for 21 minutes to observe the gradual rise in capsaicin-induced heat pain. Six 'tag-control' volumes corresponding to the rating task were removed from each scan block to limit contamination of the data. 
A reference calibration image (no labelling or background suppression, $\mathrm{TR}=6 \mathrm{~s}$, all other parameters identical to pCASL scan) was also collected to enable the estimation of the equilibrium magnetization of blood. A second calibration image of the same prescription was collected using the body coil for signal detection. This body coil calibration scan was used to correct the pCASL data for the uneven sensitivity profile of the 32-channel head coil. A T1-weighted structural image was acquired for tissue segmentation and registration purposes. We additionally acquired B0 field map images to correct for any EPI distortion effects.

\section{Pre-quantification data processing}

Analysis of perfusion imaging data was performed using FMRIB Software Library (FSL) tools (18). All raw ASL data collected from each subject were stripped of non-brain structures and motion corrected before pairwise subtraction of 'tag' and 'control' images was performed to generate perfusion-weighted images at each inversion time.

\section{Quantification of cerebral blood flow (CBF)}

All related data processing steps essential for quantification of CBF including tissue segmentation, estimation of equilibrium magnetization of blood $\left(\mathrm{M}_{0 \mathrm{~b}}\right)$ from the mean cerebrospinal fluid (CSF) magnetization $\left(\mathrm{M}_{0 \mathrm{csf}}\right)$ images, and generation of absolute $\mathrm{CBF}$ in physiological units ( $\mathrm{ml}$ blood/100g tissue/60 seconds) were completed using FSL tools. For a detailed explanation of the processing performed to obtain quantification of $\mathrm{CBF}, \mathrm{CBF}$ uncertainty and arterial arrival time (AAT) from the pCASL data, please refer to reference (17). Briefly, quantification of absolute blood flow was performed by estimation of the equilibrium magnetization of arterial blood. Voxel-wise concentration-time curves from the perfusion -weighted images were fitted to the general kinetic model to estimate both CBF and AAT (19). Perfusion parameters and goodness of fit estimates were quantified using multicomponent modeling with a Bayesian inference tool (BASIL) developed for this purpose (http://fsl.fmrib.ox.ac.uk/fsl/fslwiki/BASIL) (20). Importantly, these parameters were estimated for each complete set of inversion times, thus generating a time series of absolute CBF from each phase of the experimental paradigm. The variational Bayes approach implemented by BASIL and physiological fitting parameters used have been described previously $(17,20)$.

\section{Post-quantification processing}

The time-series of epochs generated for each scan was averaged using a mixed effects model, which accounted for the voxel-wise variance of the Bayesian fit. This generated a single voxelwise $\mathrm{CBF}$ image for each scan, with a corresponding variance image. Quantified images were transformed to standard MNI space for the comparisons described below. All images are in radiological convention.

\section{Statistical analysis}

Statistical analyses were performed using IBM SPSS Statistics, version 18 (IBM, Armonk, NY, USA). A minimum sample size of 12 was estimated following a power calculation using data from a previously published study (Ref. 17 Mezue et al 2014) on this technique 
collected from healthy adult human participants-power of $80 \%, \mathrm{p}<0.05,7.5 \%$ signal change estimated from variance in a bilateral secondary somatosensory anatomical mask. This is in line with other ASL and BOLD studies which commonly necessitate an $n=12$ to achieve $80 \%$ power at a single voxel level for typical activations when using a liberal threshold of 0.05. Imaging and psychophysical data was normally distributed (Shapiro-Wilk test: $\mathrm{p}>0.05$ ). Data collection and analysis did not require blinding to the conditions of the experiment. As described above, pain ratings were collected using a digitized, preprogrammed visual analogue scale (VAS) to reduce potential sources of bias and experimental confounds related to 'experimenter-participant' dialogue.

\section{Psychophysics}

A one-way ANOVA was used to assess the effect of time on pain ratings during the 'pain onset' period. Corresponding pairwise t-tests were performed to compare pain ratings at each time point with zero (e.g. the first time point). Post-hoc correction for multiple comparisons was applied using a Bonferroni correction.

\section{Analysis of absolute CBF changes with pain}

To interrogate regions exhibiting shared covariance between absolute perfusion changes and pain ratings, a linear regression was performed between each subject's absolute CBF time course and the corresponding pain ratings over the entire experiment. The corresponding subject-specific statistical maps were averaged using a mixed effects model. To identify regions correlated to pain perception, we used a cluster correction method at a $\mathrm{Z}$ threshold of 2.3 and significance of $p<0.01$. For illustration purposes, ASL time-courses were extracted from the resulting cluster displayed in Figure 2a (after applying the Harvard-Oxford anatomical mask for the insula cortex in FSLview to ensure anatomical specificity). Here, in Figure $2 \mathrm{~b}$ we plotted the relationship between the group mean pain intensity ratings and the mean absolute CBF changes extracted from the peak active dpIns cluster shown in Figure 2a. This is further demonstrated by the time course plotted in Figure 2c in which the group mean pain intensity ratings (grey) and the group mean absolute CBF extracted from the peak dpIns cluster are plotted over time for the full pain experiment.

\section{Exploration of dpIns involvement in the perception of pain: examples from direct cortical stimulation and acute pain somatotopy studies}

To compare the main result of the study displayed in Figure 2 with other published studies on the role of the dpIns in pain, the following analysis was conducted. Spherical functional masks for the dorsal posterior insula were generated using previously reported locations of activated clusters linked to the perception of pain by subjects receiving either direct cortical stimulation of this region or by acute stimulation of different peripheral body sites.

Activation clusters in BLUE and PURPLE represent activation clusters triggered by the acute painful stimulation of subject's feet (laser stimulation; ref 11, Baumgartner, U. et al. 2010 \& heat stimulation; ref 9, Brooks, JCW et al. 2005). The activation clusters in RED and YELLOW represent surgical coordinates at which direct electrical stimulation there resulted in the perception of pain at a particular body site (red = face pain; yellow = lower limb pain) (ref 12, Mazzola, L. et al. 2009). The spherical cluster in GREEN represents the main finding of the current study [i.e. cluster centred at the peak zstat displayed in Figure 2: 
$(-34,-20,18)]$. For clarity, spherical masks were generated using FSLtools where the size of each sphere was defined by the magnitude of coordinate ranges $\pm(x, y, z)$ reported by each study projected onto the contralateral (i.e. left) hemisphere and are displayed in Figure 3.

\section{Perfusion changes during the 'peak pain' relative to baseline}

We further analyzed the whole brain response to the peak capsaicin pain period (7 min) using paired t-test (i.e. peak pain vs baseline) in FEAT. Here, a paired t-test was performed between the baseline and 'pain peak' block using a mixed effects linear model. Statistical significance was determined by performing cluster correction at a $\mathrm{Z}$ threshold of 2.3 and significance of $\mathrm{p}<0.05$ (Supplementary figure 1).

\section{Investigation of the neural correlates of innocuous, ongoing vibratory stimulation}

To test the specificity of the dpIns result reported in the current study, we repeated a modified version of Phase I of the experimental design using a non-noxious tonic vibration stimulus applied to the subject's right foot. After the initial baseline scan (i.e. vibration OFF), the vibration stimulus was applied for approximately 7 minutes. To minimize the effects of rapid habituation to the tonic vibration, the stimulus frequency was oscillated between $1.0 \& 2.0 \mathrm{~Hz}$ (Amplitude 1mA) at pseudo-random intervals between 30-90 seconds. To test if the dpIns result presented previously was specific to the tonic heat pain, we used the same analysis procedure presented in the methods section above. Briefly, we conducted a linear regression analysis between the whole brain absolute $\mathrm{CBF}$ and: i) the ongoing stimulus frequency changes applied; and separately, ii) the ongoing vibration intensity ratings (Mixed Effects, $\mathrm{z}>2.3$, $\mathrm{p}<0.05$; cluster corrected; $\mathrm{n}=12$ ) (Supplementary figure 2).

\section{Supplementary Material}

Refer to Web version on PubMed Central for supplementary material.

\section{References}

1. Rainville P, et al. Science. 1997; 277:968-71. [PubMed: 9252330]

2. Ploghaus A, et al. Science. 1999; 284:1979-81. [PubMed: 10373114]

3. Wiech K, et al. J Neuroscience. 2010; 48:16324-31.

4. Wager TD, et al. N Engl J Med. 2013; 15:1388-97.

5. Denk F, McMahon SB, Tracey I. Nat Neuroscience. 2014; 2:192-200.

6. Mouraux A, et al. Neuroimage. 2011; 54(3):2237-49. [PubMed: 20932917]

7. Craig AD. J Comp Neurol. 2013; 1:36-63.

8. Evrard HD, Logothetis NK, Craig AD. J Comp Neurol. 2014; 522:64-97. [PubMed: 23900781]

9. Brooks JC, et al. Neuroimage. 2005; 1:201-9.

10. Henderson LA, et al. Pain. 2007; 128:20-30. [PubMed: 17011704]

11. Baumgärtner U, et al. J or Neurophysiol. 2010; 104:2863-72.

12. Mazzola L, et al. Pain. 2009; 146:99-104. [PubMed: 19665303]

13. Garcia-Larrea L, et al. Brain. 2010; 133:2528-39. [PubMed: 20724291]

14. Greenspan JD, Lee RR, Lenz FA. Pain. 1999; 3:273-82.

15. Garcia-Larrea L, Peyron R. Pain. 2013; 154:S29-S43. [PubMed: 24021862]

16. Baliki MN, et al. Nat Neurosci. 2012; 15:1117-9. [PubMed: 22751038] 
17. Mezue M, Segerdahl AR, et al. J Cereb Blood Flow Metab. 2014; 34:1919-27. [PubMed: 25269517]

18. Smith SM, et al. Neuroimage. 2004; 23(suppl 1):S208-S219. [PubMed: 15501092]

19. Buxton R, Frank L. J Cereb Blood Flow Metab. 1997; 17:64-72. [PubMed: 8978388]

20. Chappell M, et al. IEEE Trans Sign Proc. 2009; 57:223-236. 


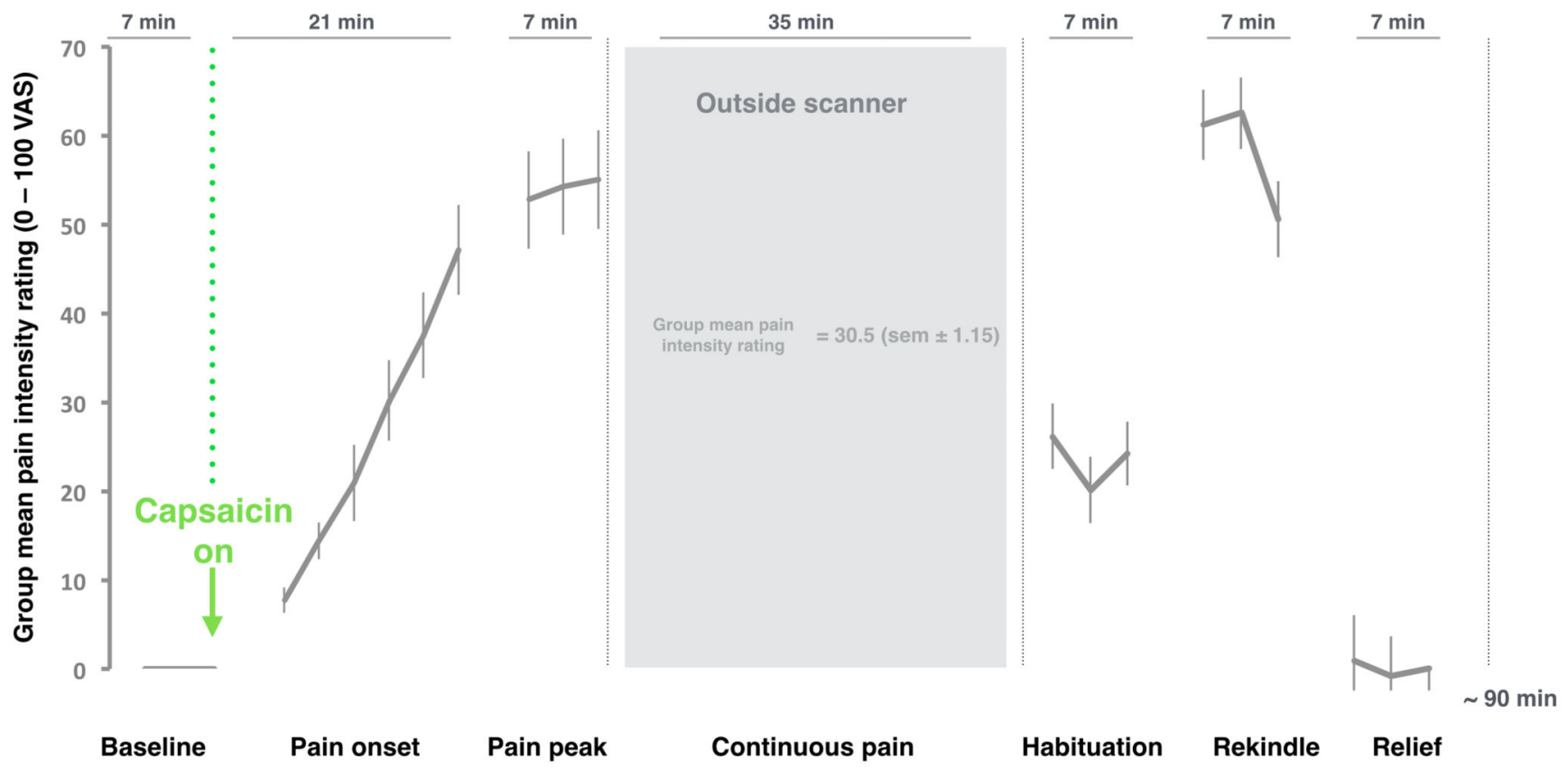

Figure 1. Tonic pain ratings over time.

Group mean pain intensity ratings (grey) are plotted over time (x-axis). Error bars represent the standard error of the mean (s.e.m.). Results from the repeat-measures ANOVA are in supplementary table 1 . 

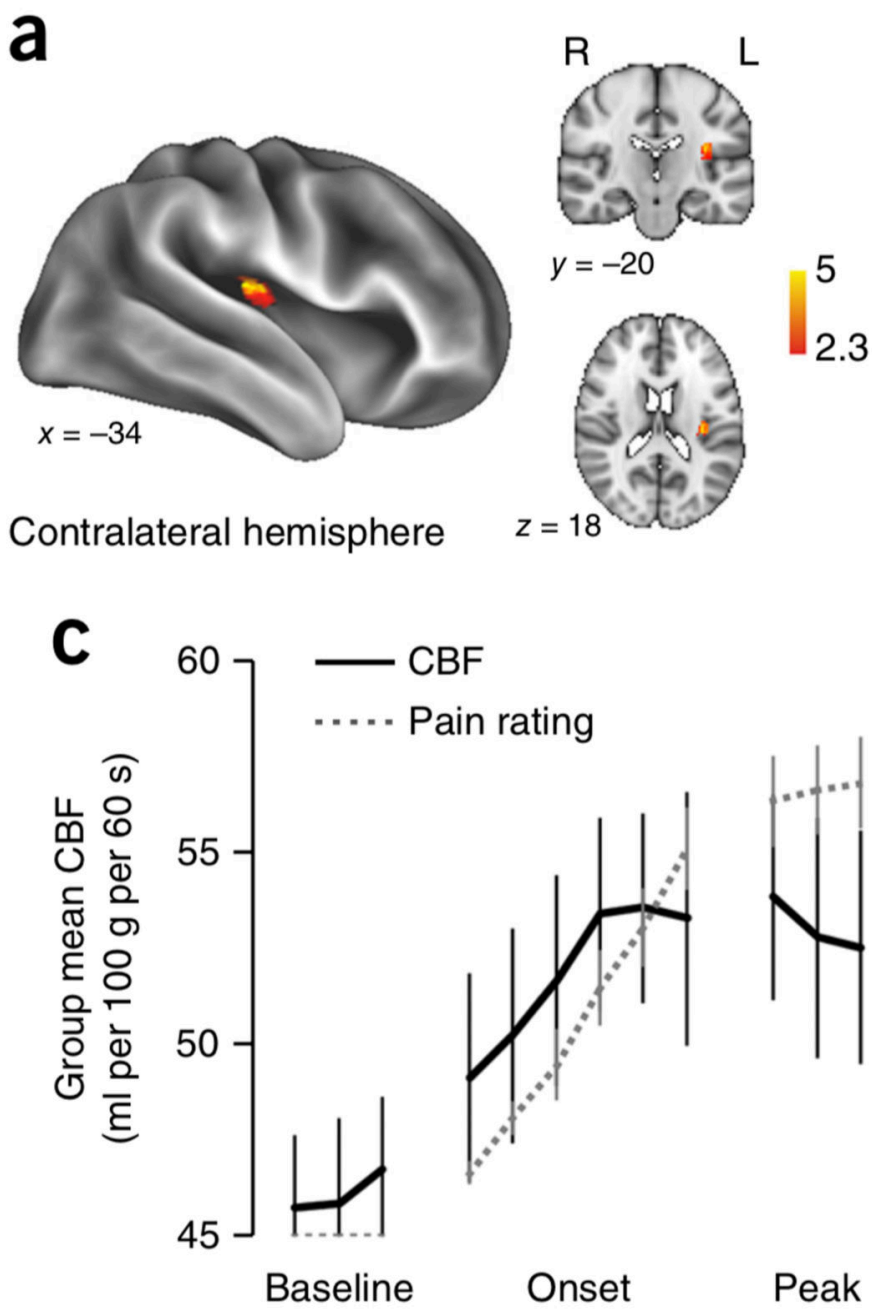

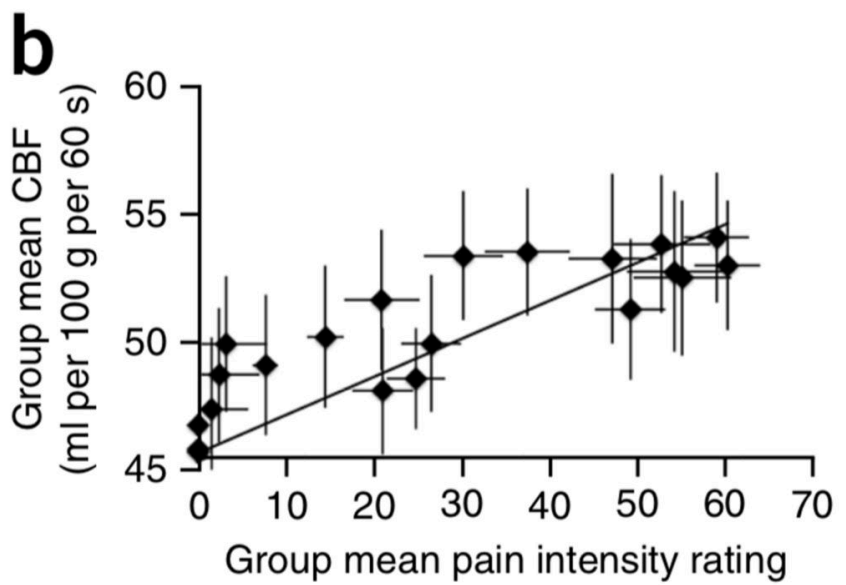

(0-100 VAS)
Peak

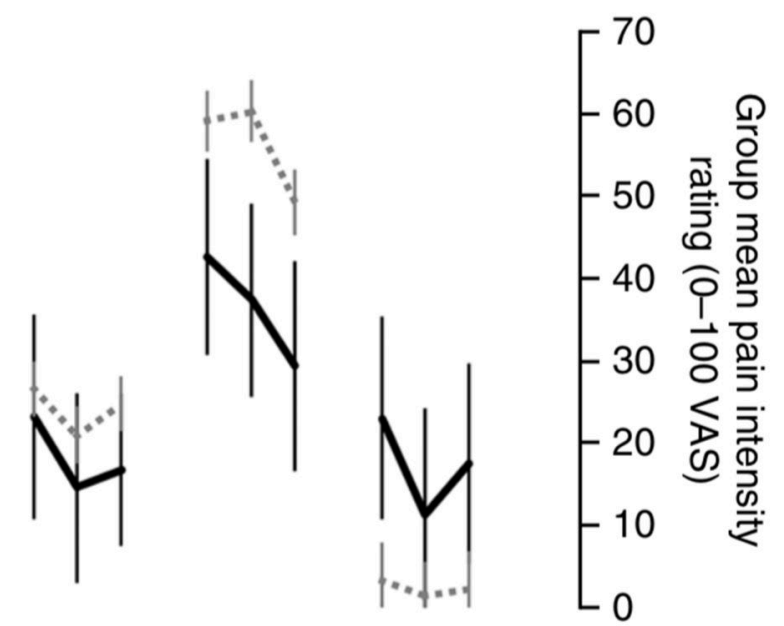

Hab. Rekindle Relief

Figure 2. Whole brain absolute $\mathrm{CBF}$ correlation with pain ratings.

a) Contralateral dpIns shows a strong correlation between absolute CBF and pain ratings. Voxels with supra-threshold activation are shown in red (Linear regression; Mixed Effects, $\mathrm{z}>2.3$; $\mathrm{p}<0.01$ ). Radiological convention is used (L: left; R: right). b) A plot of the group mean tonic pain ratings versus the absolute $\mathrm{CBF}$ in the contralateral dpIns. c) Group mean absolute CBF extracted from the peak contralateral dpIns cluster alongside the ongoing pain intensity ratings (grey) over time. Error bars represent the sem. Further analysis of this data set exploring more conventional contrasts of "Peak" period only minus "Baseline" period can be found in Supplementary Information, Supplementary figure 1 and Supplementary table 2 . 


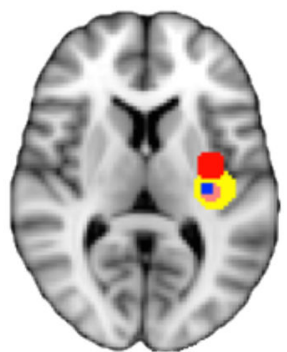

$z=8$

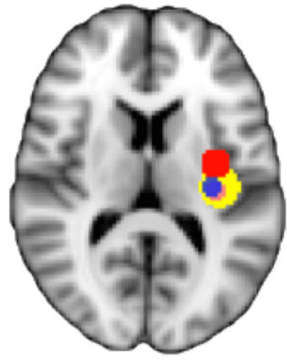

$z=12$

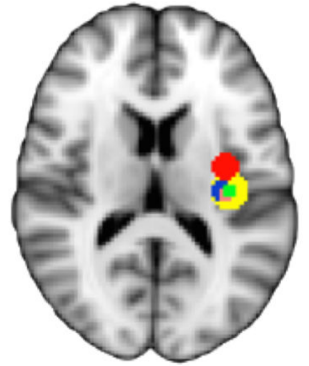

$z=14$

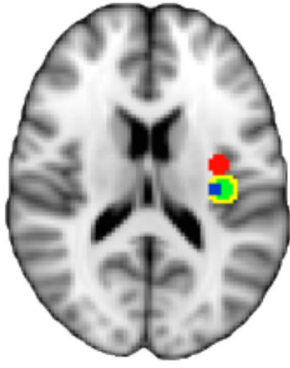

$z=16$

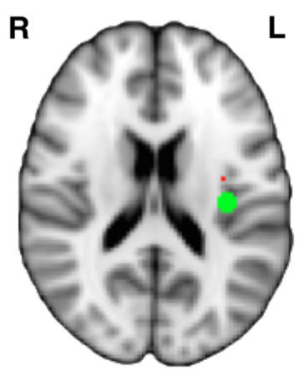

$z=18$

\section{Direct electrical stimulation of posterior insula:}

Mazzola, L. et al. (2009)

Face pain: $(-35 \pm 4,-6.4 \pm 6,11 \pm 8)$

Lower limb pain: $(-36 \pm 5,-19 \pm 11,8 \pm 3)$
Tracking ongoing tonic heat pain: Current study

Peak active cluster: $(-34,-20,18)$

\section{Acute pain somatotopy of posterior insula: \\ Brooks, JCW. et al. (2005) \\ Thermal stimulus applied to foot: $(-35 \pm 4,-20.8 \pm 6,11 \pm 5)$ \\ Baumgärtner, U. et al. (2010) \\ Laser stimulus applied to foot: $(-32 \pm 2,-20 \pm 1.8,12 \pm 2.4)$}

Figure 3. A schematic of dpIns involvement in human pain studies.

Spherical functional masks were generated from previously reported MNI coordinates linked to the perception of pain. Activation clusters in BLUE and PURPLE represent activation clusters triggered by acute painful stimulation of subjects' feet using heat (9) or a laser (11). The activation clusters in RED and YELLOW represent surgical coordinates at which direct electrical stimulation resulted in the perception of pain at a particular body site (red = face pain; yellow = lower limb pain) (12). For clarity, the spherical cluster centered at peak zstat reported in the current study is displayed in green. Radiological convention is used (L: left; R: right). 